\title{
Simulation and Analysis of Outdoor Microcellular Radio Propagation Characteristics Based on the Method of SBR/Image
}

\author{
Xue Ma, Yuanjian Liu, Qinjian Shi, Yerong Zhang \\ College of Electronic Science and Engineering, Nanjing University of Posts \& Telecommunications, Nanjing, China \\ Email: xuem89@126.com
}

Received January 2015

Copyright $(2015$ by authors and Scientific Research Publishing Inc. This work is licensed under the Creative Commons Attribution International License (CC BY). http://creativecommons.org/licenses/by/4.0/

\section{(c) (7) Open Access}

\begin{abstract}
In this paper, the outdoor microcellular radio propagation characteristics at $3.5 \mathrm{GHz}$ are simulated and analyzed by the method of SBR/Image (Shooting and bouncing ray tracing/Image). A good agreement is achieved between the results simulated and the results given in published literature. So the correctness of the method has been validated. Some simulated propagation parameters of LOS (Line-of-sight) and NLOS (None-line-of-sight) have been compared. The analysis of the above results provides the foundation for the coverage of outdoor microcellular systems.
\end{abstract}

\section{Keywords}

SBR/Image, Outdoor Microcell, Propagation Characteristics

\section{Introduction}

As mobile communication service has increased dramatically in recent years, the microcellular systems [1]-[5] are needed to accommodate more users with limited resource of frequency. So it is important to predict propagation characteristics (such as path loss, RMS delay spread) for better wireless coverage of outdoor microcellular systems. The ray tracing techniques are usually employed to study the radio wave propagation of outdoor microcellular environments. The several most popular ray tracing techniques are image method, brute force ray tracing, deterministic ray tube method and the method of SBR/Image. Image method [6] has good efficiency for it does not require the reception tests. However, images of scatters are difficult to find in complex environments. Brute force ray tracing [7] can be used in complex environments, but it needs reception tests. A deterministic ray 
tube method [8] saves computer resources, but it needs to create a ray tree based on the actual environment. SBR/Image method [9] can be used for complex environment, and it can find all propagation paths from the transmitter to the receiver with high accuracy and computational efficiency. So this method is a valuable method which can be applied to predict the radio wave propagation.

\section{Simulation Environment}

Figure 1 shows the top view of simulation environment in [10] which is a rectangular with dimensions $380 \times$ $180 \mathrm{~m}$. The value of the relative permittivity $\varepsilon_{r}$ and the conductivity $\sigma$ is chosen as $3,0.005 \mathrm{~S} / \mathrm{m}$ for buildings and $15,7 \mathrm{~S} / \mathrm{m}$ for the ground. In the simulation, vertically polarized omnidirectional antennas with $0 \mathrm{dBi}$ are used for both the transmitter and the receiver. The heights of transmitter antenna and receiver antenna are 25 $\mathrm{m}$ and $1.5 \mathrm{~m}$. The frequency of transmitted signal is $3.5 \mathrm{GHz}$, and the emitted power is $0 \mathrm{dBm}$. The receiver trajectories include line-of-sight (A-C-B) street, none-line-of-sight (A-C-D) street, the parallel street (E-F) and the perpendicular street $(\mathrm{G}-\mathrm{H})$.

\section{Simulation Results}

Figure 2 shows signal path loss versus distance between the transmitter and the receiver. A good agreement is achieved between the results simulated and the results given in literature [10], so the correctness of our method has been validated. It is found that signal path loss increases as distance increases, and it rapidly increases when the receiver moves along NLOS street, because there is no direct ray and the diffracted rays are definitely dominant.

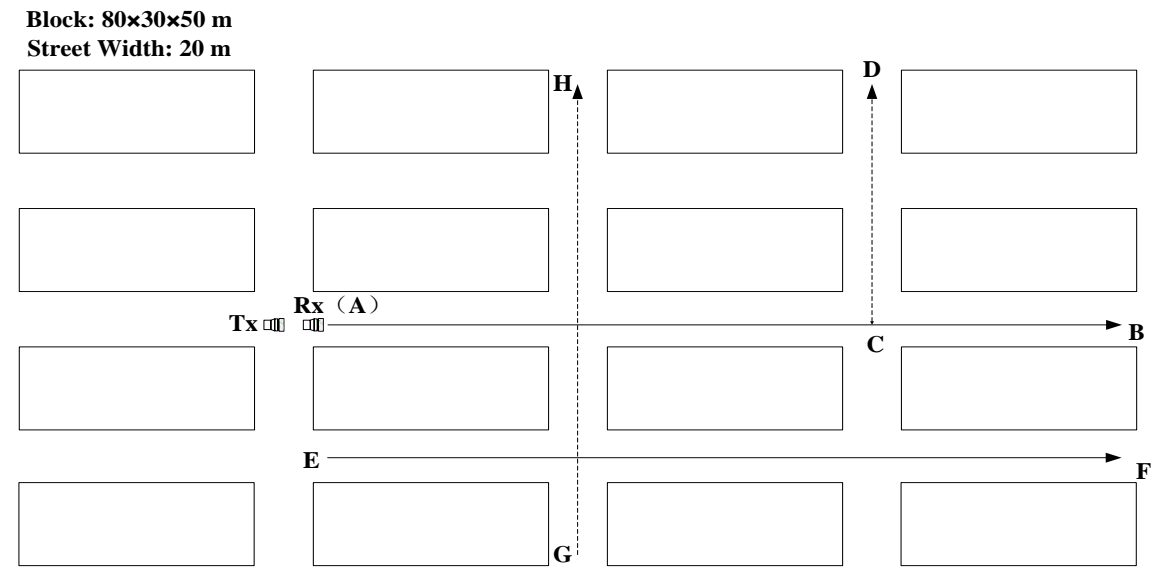

Figure 1. Top view of simulated environment.

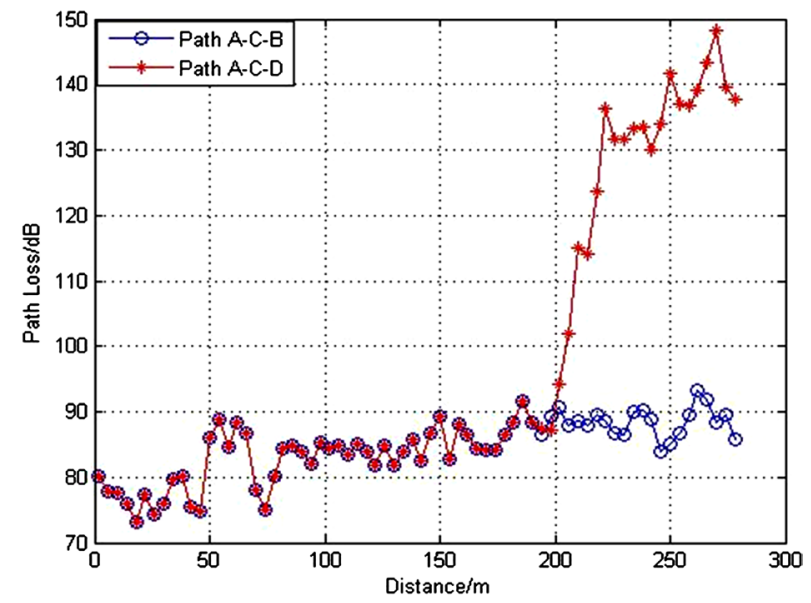

Figure 2. Simulated signal path loss. 
When a receiver moves along the LOS street A-C-B, the NLOS street A-C-D, the parallel street E-F and the perpendicular street G-H, the path loss and the RMS delay spread are presented in Figure 3 and Figure 4, respectively. In Figure 3, the path loss of LOS path shows the lowest decay because the direct ray is dominant. In the street A-C-D, signal path loss increases rapidly when the receiver runs into the NLOS region (C-D). The path loss also increases as distance increases when the receiver moves along the parallel street (E-F). However, it is easily found that the path loss is much larger than that of the LOS path. The plotted path loss curves of streets both A-C-D and E-F are seen to agree closely for distance greater than $200 \mathrm{~m}$. In Figure 3, the curve of street G-H is first down and then up, an obvious decrease of the path loss is observed when the receiver moves toward the crossroad.

The RMS delay spread is the square root of the second central moment of power delay profile. It is an important parameter to characterize wide-band multipath channels. In this paper, the RMS delay spread of four paths is plotted in Figure 4. The delay spread of LOS path is below $0.2 \mu \mathrm{s}$. It is seen from Figure 4 that the delay

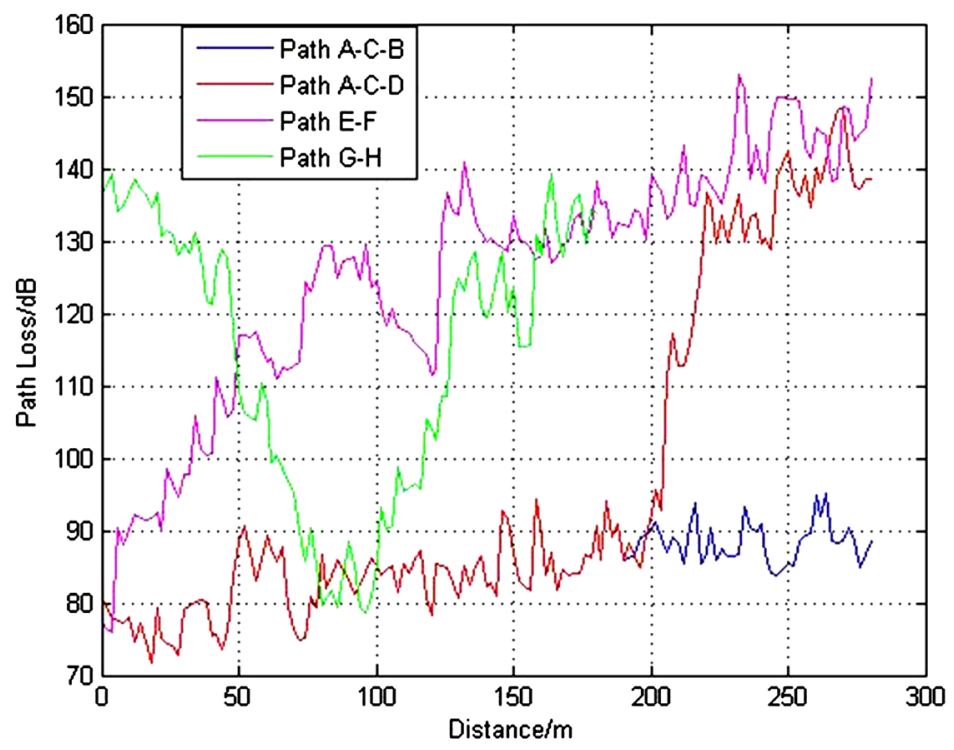

Figure 3. The comparison of the path loss of four streets.

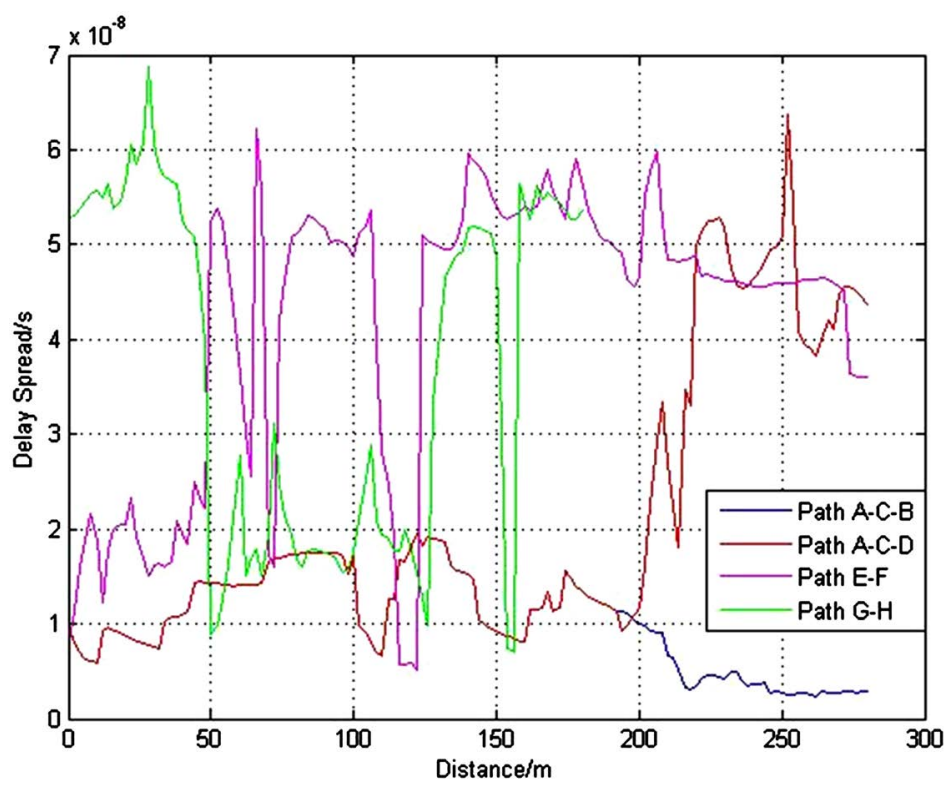

Figure 4. The comparison of the delay spread of four streets. 
spread of the NLOS path increases rapidly compared with LOS path, which means intersymbol interference (ISI) is larger than that of LOS region. When the receiver moves along the parallel street (E-F) and the perpendicular street $(\mathrm{G}-\mathrm{H})$, the delay spread presents sharp fluctuation for more obstructions hinder the radio wave reaching to the receiver compared to the LOS street.

The formula of the Doppler shift is $\Delta f=f_{0}\left[\frac{\bar{d} \cdot \overline{V_{T}}+\bar{a} \cdot \overline{V_{R}}}{c}\right]$. So each path will cause the Doppler shift when the transmitter or the receiver is moving. Therefore, the Doppler shift can be determined (Assuming that the transmitter is fixed and the speed of receiver is $1 \mathrm{~m} / \mathrm{s}$ ). The comparison of the Doppler shift when the receiver moves along four streets are shown in Figure 5. The Doppler shift of LOS path is very flat and its value is lower than $-10 \mathrm{~Hz}$. In the path A-C-D, the Doppler shift presents severe oscillation when the receiver runs into the NLOS path (C-D) because there are more diffraction rays rather than direct ray. It varies between $-10 \mathrm{~Hz}$ and 0 Hz. The Doppler shift of the parallel street E-F shows sharp fluctuation for distance lower than $125 \mathrm{~m}$. As distance increases, the curve becomes flat for little variation of mean direction of arrival. The values of Doppler shift in path A-C-B, path A-C-D and path E-F are all minus because the receiver runs away from the transmitter. The Doppler shift of the perpendicular street G-H shows severe oscillation and has positive number. It varies between $-15 \mathrm{~Hz}$ and $10 \mathrm{~Hz}$. The range of Doppler shift in this simulation provides the theoretical foundation for the coverage of outdoor microcellular systems.

The angles $\theta_{A}$ and $\phi_{A}$ give the direction from which the propagation path arrives at a receiver point. Therefore, the direction of arrival is given by the unit vector as

$$
\bar{a}=\sin \left(\theta_{A}\right) \cos \left(\phi_{A}\right) \bar{x}+\sin \left(\theta_{A}\right) \sin \left(\phi_{A}\right) \bar{y}+\cos \left(\phi_{A}\right) \bar{z} .
$$

The angle $\bar{\theta}$ is defined as $\bar{\theta}=\tan ^{-1}\left(\frac{\sqrt{A_{x}^{2}+A_{y}^{2}}}{A_{z}}\right)$, and the angle $\bar{\phi}$ is defined as $\bar{\phi}=\cos ^{-1}\left(\frac{A_{y}}{A_{x}}\right) \quad\left(A=\sum_{i=1}^{N} P_{i} \bar{a}\right)$.

Figure 6 and Figure 7 show the distribution of mean angle of arrival (including angles $\bar{\theta}$ and $\bar{\phi}$ ) of all received points at two paths (the LOS path A-C-B and the perpendicular path G-H). In Figure 6(a), the mean angle of arrival $(\bar{\phi})$ in path A-C-B varies between $177^{\circ}$ and $182^{\circ}$ and it distributes around $180^{\circ}$. The corresponding angle $\bar{\phi}$ in path G-H is shown as Figure 6(b). It varies between $27^{\circ}$ and $334^{\circ}$ and has a wider variation range compared with the mean angle of arrival in path A-C-B. In Figure 7(a), the mean angle of arrival $(\bar{\theta})$ in path A-C-B varies between $58^{\circ}$ and $87^{\circ}$ and it distributes around $85^{\circ}$. The angle $\bar{\theta}$ in path G-H is shown as Figure 7(b). It varies between $79^{\circ}$ and $87^{\circ}$ and it distributes around $83^{\circ}$. The distribution of mean angle of arrival $(\bar{\theta})$ has little difference between the NOS path A-C-B and the perpendicular path G-H.

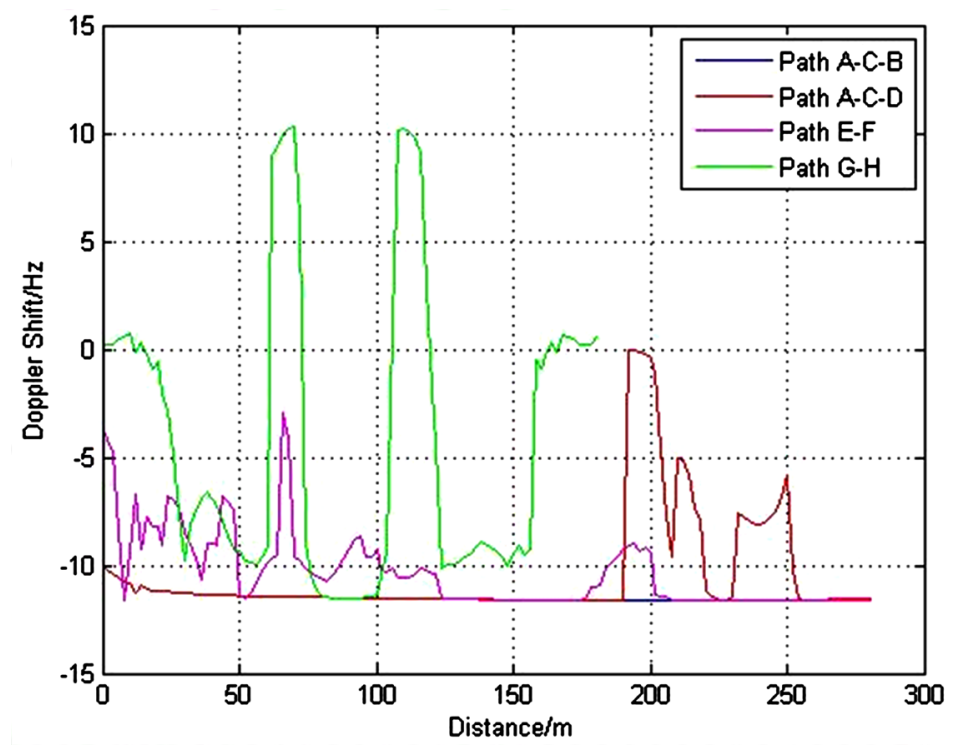

Figure 5. The comparison of the Doppler shift of four streets. 


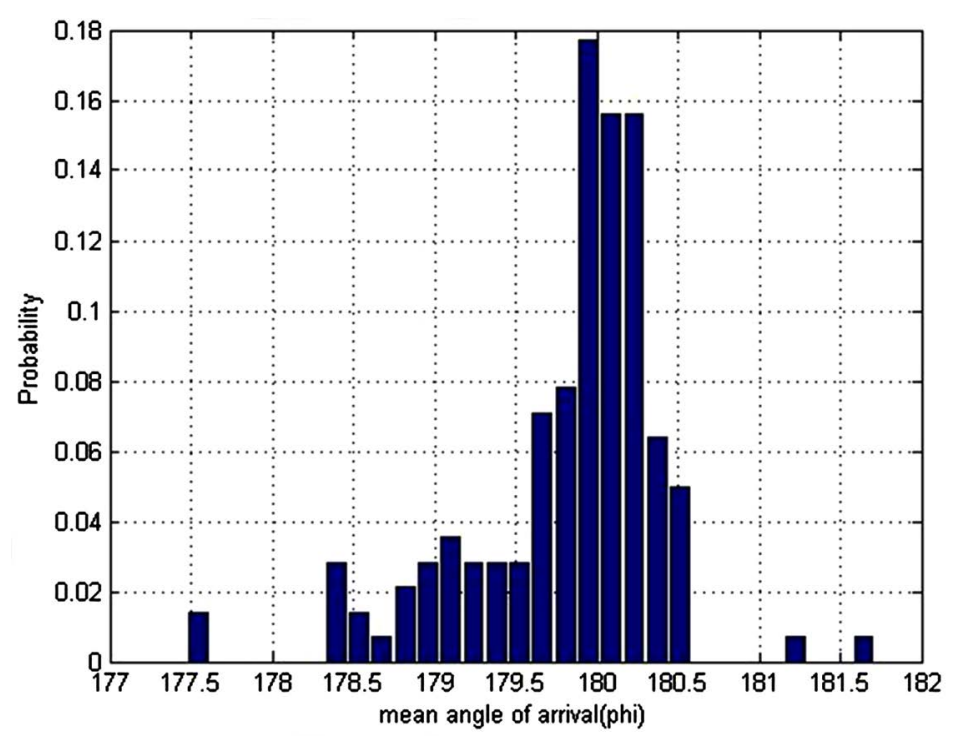

(a)

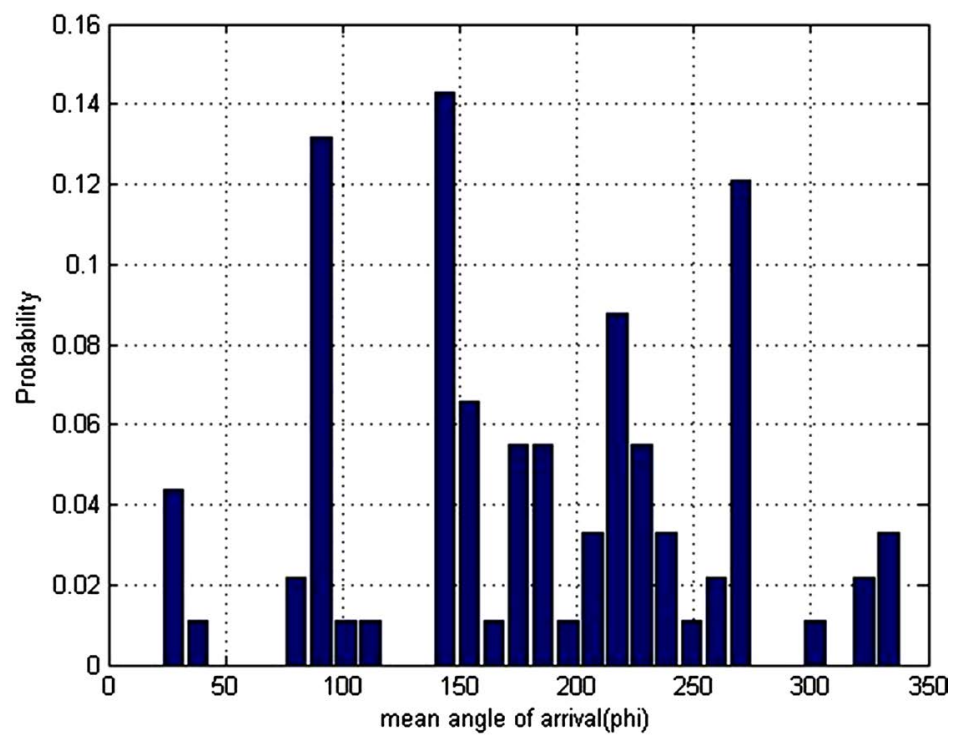

(b)

Figure 6. Distribution of mean of arrival $(\bar{\phi})$ : (a) path A-C-B; (b) path G-H.

\section{Conclusion}

In this paper, the method of SBR/Image is employed to study the radio wave propagation in outdoor microcellular environment at $3.5 \mathrm{GHz}$. The simulated results show good agreement with the results in the literature, so the correctness of the method has been validated. The path loss curve in LOS path is flat and increases slowly versus distance, the corresponding path loss in NLOS street shows much higher attenuation. The delay spread of NLOS street presents sharp fluctuation compared with that of LOS path, which means intersymbol interference (ISI) strengthen. The value of Doppler shift of path A-C-B, path A-C-D and path E-F are all minus because the receiver runs away from the transmitter. In the perpendicular street $\mathrm{G}-\mathrm{H}$, Doppler shift shows severe oscillation and has positive number. The mean angle of arrival $(\bar{\phi})$ in perpendicular path G-H has a wider variation range compared with that in NOS path A-C-B. The distribution of mean angle of arrival $(\bar{\theta})$ has little difference between the NOS path A-C-B and the perpendicular path G-H. The analysis of the above results provides the theoretical foundation for the coverage of outdoor microcellular systems. 


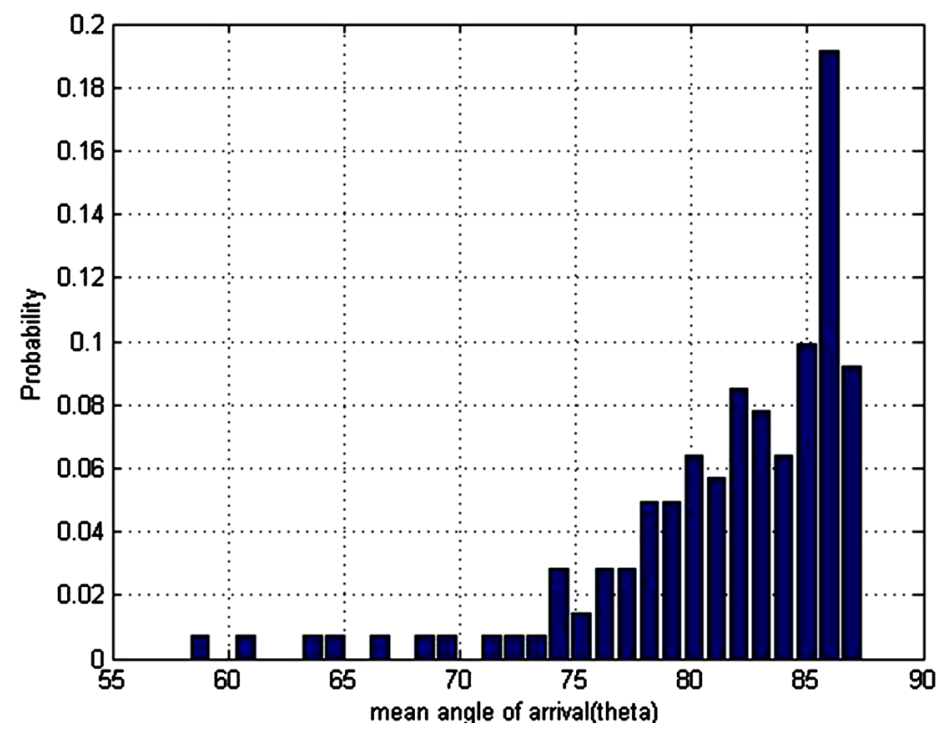

(a)

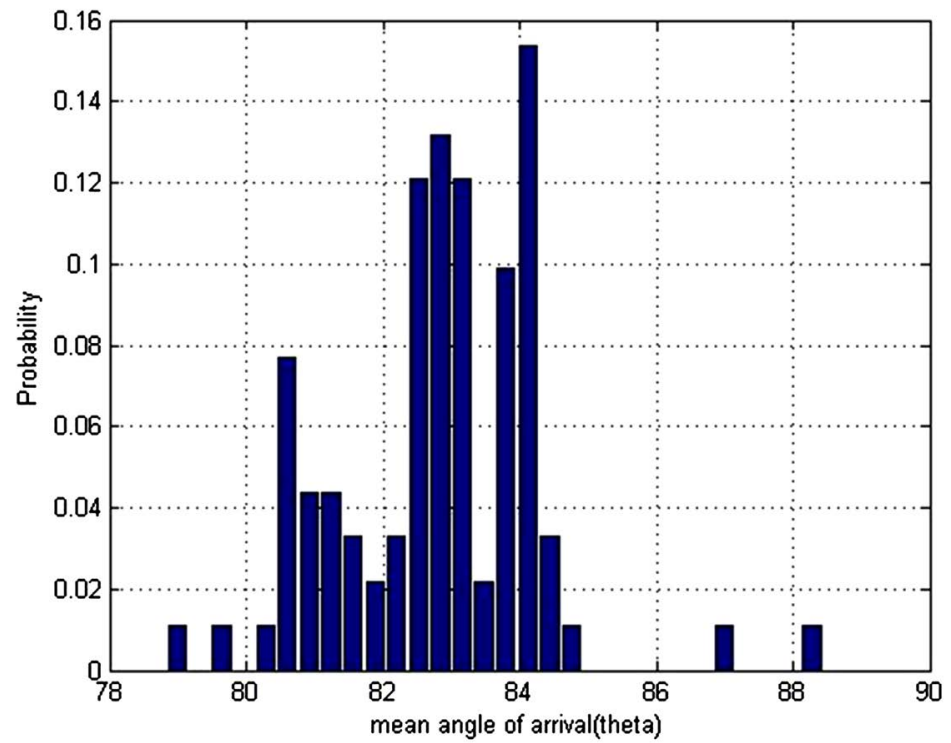

(b)

Figure 7. Distribution of mean of arrival ( $\bar{\theta}$ ): (a) path A-C-B; (b) path G-H.

\section{Acknowledgements}

This work has been supported by National Natural Science Fund under Grant no.61372045 and by the Ministry of Education of Higher Specialized Research Fund for the Doctoral Program under Grant no.20123223120003.

\section{References}

[1] Kitao, K. and Ichitsubo, S. (2004) Path Loss Prediction Formula for Microcell in $400 \mathrm{MHz}$ to 8 GHz Band. Electronics Letters, 40, 685-687. http://dx.doi.org/10.1049/el:20040475

[2] Walden, M.C. and Rowsell, F.J. (2005) Urban Propagation Measurements and Statistical Path Loss Model at 3.5GHz. IEEE Antennas and Propagation Society International Symposium, 2005, 1A:363-366.

[3] Xia, H.H., Bertoni, H.L., Macicel, L.R., Stewart, A.L. and Rowe, R. (1993) Radio Propagation Charateristics for Lineof-Sight Microcellular and Personal Communications. IEEE Trans. on Antennas and Propagation, 41, 1439-1447.

[4] Blaunstein, N.R. and Levin, M. (1998) Characteristics’ Prediction in Urban and Suburban Environments. IEEE Trans- 
actions on Vehicular Technology, 47, 225-234. http://dx.doi.org/10.1109/25.661049

[5] Tan, S.Y. and Tan, H.S. (1996) A Microcellular Communications Propagation Model Based on the Uniform Theory of Diffraction and Multiple Image Theory. IEEE Transactions on Antennas and Propagation, 44, 1317-1326. http://dx.doi.org/10.1109/8.537325

[6] Grubisic, S. (2006) Ray-Tracing Propagation Model Using Image Theory with a New Accurate Approximation for Transmitted Rays Through Walls. IEEE Transactions on Antennas and Propagation, 42, 835-838.

[7] Seidel, S.Y. and Rappaport, T.S. (1994) Site-specific Propagation Prediction for Wireless in-Building Personal Communication System Design. IEEE Transactions on Vehicular Technology, 43, 879-891. http://dx.doi.org/10.1109/25.330150

[8] Son, H.W. and Myung, N.H. (1999) A Deterministic Ray Tube Method for Microcellular Wave Propagation Prediction Model. IEEE Transactions on Antennas and Propagation, 47, 1344-1350. http://dx.doi.org/10.1109/8.791954

[9] Chen, S.H. and Jeng, S.K. (1996) SBR Image Approach for Radio Wave Propagation in Tunnels with and without Traffic. IEEE Transactions on Antennas and Propagation, 3, 570-578.

[10] Wang, Z.B., Li, G.H., Zhang, J., Yan, J. and Wang, H.-W. (2009) Research of Radio Propagation Characteristics at 3.5 GHz in Outdoor Environment. Modern Electronics Technique, 308, 63-66. 